\title{
Inflammatory Bowel Disease Phenotype in Pediatric Primary Sclerosing Cholangitis
}

\author{
Laura Lascurain, MD*, Micheal Kyle Jensen, MD*, Stephen L. Guthery, MD*, John Holmen, \\ $\mathrm{PhD}^{\dagger}$, and Mark Deneau, MD* \\ *Department of Pediatrics, University of Utah, Salt Lake City, Utah \\ tHomer Warner Center for Information Research, Intermountain Healthcare Corporation, Salt \\ Lake City, Utah.
}

\section{Abstract}

Background: Primary sclerosing cholangitis (PSC) is a chronic cholestatic liver disease that most often occurs in association with inflammatory bowel disease (IBD). We examined whether the activity or colonic distribution of IBD differed in pediatric patients with and without PSC.

Methods: We compared colonic disease distribution, physician global assessment scores, Mayo endoscopic severity scores, IBD-related hospital admissions, and colonic resection surgery rate in a retrospective cohort of pediatric patients with IBD with and without PSC.

Results: We identified 37 patients with PSC-IBD, and 137 non-PSC matched IBD controls. Pancolitis was seen in 89.7 versus $72.4 \%(P=0.051)$ of patients with PSC-IBD and rectal sparing in 24.3 versus $21.6 \%(P=0.721)$ of patients with IBD. Physician global assessment and Mayo scores at presentation and in follow-up were similar in PSC-IBD and IBD. Patients with PSC-IBD had 0.19 admissions per person-year compared with 0.25 in patients with IBD. The incidence rate ratio for admission was 0.75 (95\% confidence interval (CI), 0.51-1.08). The 5-year probability of colonic surgery was $16.4 \%$ (95\% CI, 7.0-36.0) in patients with PSC-IBD and $24.7 \%$ (95\% CI, 17.7-33.8) in patients with IBD $(P=0.271)$. In a multivariate model, male sex (hazard ratio [HR] $=2.2[95 \% \mathrm{CI}, 1.1-4.3])$ and the presence of a non-PSC immune-mediated comorbidity $\{\mathrm{HR}=3.9$ (95\% CI, 1.5-10.4), but not PSC (HR $=0.5[95 \%$ CI, 0.2-1.3]) $\}$ or Crohn's disease (HR $=0.5$ [95\% CI, 0.1-1.5]), were risk factors for colonic surgery in pediatric IBD.

Conclusions: Patients with IBD and PSC were more likely to present with pancolitis, but had similar rates of rectal sparing. Patients with IBD showed similar disease activity across a wide range of measures, at presentation and in follow-up, regardless of the presence of PSC.

\section{Keywords}

pediatric; cholangitis; sclerosing; colonoscopy; Crohn's disease; ulcerative colitis; inflammatory bowel disease; colectomy

Reprints: Mark Deneau, MD, Department of Pediatrics, University of Utah, 81 N. Mario Capecchi Drive, Salt Lake City, UT 84113 (mark.deneau@hsc.utah.edu).

The authors have no conflict of interest to disclose. 
Primary sclerosing cholangitis (PSC) is a chronic cholestatic liver disease characterized by progressive fibrosis and obliteration of intrahepatic bile ducts and extrahepatic bile ducts. ${ }^{1}$ Most cases of PSC occur in individuals with inflammatory bowel disease (IBD). The IBD in patients with PSC has been described as a unique phenotype (PSC-IBD) with activity and distribution that is distinct from isolated ulcerative colitis (UC) and Crohn's disease (CD). ${ }^{2}$ PSC-IBD has mainly been described in adult populations and is characterized by a rectal sparing, pancolonic distribution with frequent backwash ileitis, mild inflammation, mild disease activity, and a greater risk of colorectal neoplasia. ${ }^{3}$ These features of PSC-IBD have been conflicting however, ${ }^{4}$ and there is a paucity of data on PSC-IBD in children to date. The objective of this study was to characterize and compare the distribution and activity of IBD in pediatric patients with and without PSC using a population-based cohort.

\section{METHODS}

The state of Utah provides a unique opportunity to study pediatric intestinal and liver disease in a population-based fashion. All pediatric gastroenterologists, all adult hepatologists, all 3 tertiary referral hospitals, and all 3 liver transplant programs in a geographically isolated region of the Western United States reside within 2 large health care organizations. We previously identified all cases of IBD, PSC, and autoimmune sclerosing cholangitis diagnosed before 18 years of age between January 1, 2005 and December 31, 2011 in the state of Utah by reviewing medical records from both health care organizations. ${ }^{5}$ We used a retrospective cohort design and matched each PSC-IBD case to 4 patients with non-PSC IBD who had the same disease phenotype (UC versus CD). We excluded 11 cases that lacked sufficient records for disease activity scoring at diagnosis.

We retrospectively reviewed medical records, endoscopic images, operative summaries, and histopathology reports on all patients. All research activities were approved by the institutional review boards of both Intermountain Healthcare and the University of Utah. An IBD diagnosis was based on established criteria requiring symptom chronicity, exclusion of enteric infections, and objective evidence of chronic inflammation on endoscopy and histology. ${ }^{6}$ We defined PSC as the presence of a cholestatic biochemical profile with either cholangiography showing multifocal strictures and segmental dilations or liver histology showing fibro-obliterative cholangitis or findings consistent with primary ductular involvement. ${ }^{1,7,8}$ All patients with autoimmune sclerosing cholangitis met individual diagnostic criteria for both autoimmune hepatitis (AIH) and PSC and were included in the study.

Disease distribution was assessed endoscopically and histologically at the first diagnostic endoscopy. Pancolitis was defined as the presence of histologic or endoscopic disease involving the sigmoid colon and extending proximal to the splenic flexure. Rectal sparing was defined as the absence of endoscopic and histologic disease in the rectum. Endoscopic disease activity was assessed using the Mayo endoscopic score in all patients with UC. ${ }^{9}$ Clinical disease activity was scored by the physician's global assessment (PGA) of IBD activity ${ }^{10}$ in patients with both UC and CD. Scoring was done at diagnosis and at the first clinical encounter and/or follow-up endoscopy falling between 12 to 18 months after diagnosis, if available. 
We created a retrospective cohort of all patients with PSC-IBD and IBD and followed them from the date of IBD diagnosis to the date of colonic surgery. Colonic surgery was defined as colectomy or hemicolectomy for patients with UC or resection of at least the cecum and ascending colon in patients with $\mathrm{CD}$ (i.e., more extensive than an isolated ileocectomy to exclude patients with structuring Crohn's disease complications and focus on refractory colonic Crohns). We counted the admissions related to IBD exacerbations during follow-up. In patients who had J-kpouch construction, we counted postoperative diagnoses of pouchitis. All observations were censored at the end of the review period April 13, 2013 or at the date of the last clinical encounter for patients who were lost to follow-up. We used the KaplanMeier method to determine the 5-year probability of IBD surgery.

We performed a Cox regression to determine whether PSC was a risk factor for colonic surgery in this cohort. We controlled for the presence of IBD phenotype (Crohn versus UC), patient sex, severe colonic disease (Mayo endoscopic score $=3$ ), and the presence of nonhepatic immune-mediated comorbidities using multivariate analysis. The proportional hazards assumption was assessed graphically. Variables were chosen a priori based on previously published associations and clinical observations of the authors.

We used the t test, rank sum, chi-square, and Fisher exact tests to analyze the data as appropriate. The logrank test was used to compare 5-year outcome probabilities. All calculations were done using Stata version 12 (Statacorp, College Station, TX).

\section{RESULTS}

We identified 37 patients with PSC-IBD, and 148 non-PSC matched IBD controls of which 11 patients were excluded for inadequate records. The initial endoscopy was incomplete or lacked enough data to determine whether pancolitis was present in 12\% (21/173). IBD distribution at diagnosis was similar between groups. Demographic and disease distribution information is detailed in Table 1. Comorbid nonhepatic immune-mediated conditions in patients with PSC-IBD included 1 case each of celiac disease, Hashimoto thyroiditis, juvenile idiopathic arthritis, and vitiligo, and in IBD without PSC included 2 cases of celiac disease, and 1 case each of juvenile idiopathic arthritis, chronic idiopathic thrombocytopenic purpura, vitiligo, multiple sclerosis, type 1 diabetes, and systemic vasculitis.

The endoscopic and clinical disease activity was similar at diagnosis and in follow-up in patients with PSC-IBD and IBD. The proportion of patients who improved, remained stable, or worsened over follow-up was similar. Clinical disease activity was compared in male and female subgroups at all timepoints, and there were no statistical differences. The number of IBD-related admissions was similar between groups. All disease activity measurements are detailed in Table 2.

The PSC-IBD group underwent 0.4 colonic surgeries per 1000 patient-years compared to 0.54 per 1000 patient-years in the IBD group $(P=0.44)$. The 5 -year probability of intestinal surgery was $16.4 \%$ (95\% confidence interval, 7.0-36.0) in patients with PSC-IBD and $24.7 \%$ (95\% confidence interval, 17.7-33.8) in patients with IBD. The Kaplan-Meier survival function is shown in Figure 1. A lower proportion of patients with PSC-IBD 
required intestinal surgery over time, but the survival curves were not statistically different, $P=0.271$. One IBD patient without PSC presented with an abdominal mass after having been lost to follow-up for several years and was ultimately diagnosed with stage IV colonic adenocarcinoma after liver wedge resection and colectomy. He died of complications of this cancer. Intestinal disease refractory to therapy was the indication for IBD surgery in all other cases. After surgery, pouchitis occurred in 100\% (6/6) of patients with PSC-IBD and in $63.8 \%(23 / 36)$ on patients with IBD, $P=0.076$.

Portal hypertension and recurring cholangitis occurred in 12/37 (32.4\%) of patients with PSC-IBD including 6/37 (16.2\%) of patients with PSC who underwent liver transplantation during follow-up. Of the 6 transplanted patients, one required colectomy at 1.2 years before transplant and 2 required colectomy at 1.8 and 8.3 years after transplant.

On regression analysis, PSC was not a risk factor for colonic surgery. Patients with male sex and/or patients who had a nonhepatic immune-mediated comorbidity were more likely to require colonic surgery. Detailed results of the regression analysis are presented in Table 3.

\section{DISCUSSION}

We compared the distribution and activity of IBD and the risk factors for colectomy in pediatric patients with and without PSC. There were 3 main findings from this study. First, the intestinal distribution of PSC-IBD was not different than IBD without PSC. Second, severity of IBD was similar in patients with PSC-IBD and IBD across a variety of objective disease activity markers. Finally, a diagnosis of PSC was not a risk factor for colonic surgery in a multivariate regression model.

\section{INTESTINAL DISEASE DISTRIBUTION}

\section{Pancolitis}

The intestinal distribution of disease was similar in patients with IBD to those with IBDPSC. Pancolitis was present in a larger proportion of patients with PSC-IBD than patients with IBD in this study. This result was marginally statistically significant, but in power analysis, there was a high chance of type II error $(B=0.49)$. Similar rates of $75 \%$ to $80 \%$ pancolitis at diagnosis of IBD have been observed in pediatric patients both with ${ }^{11}$ and without PSC. ${ }^{12}$ Pancolitis was seen in $64 \%$ of pediatric patients with IBD affected by comorbid autoimmune diseases compared with $30 \%$ with isolated IBD, ${ }^{13}$ which was different from our series. The authors analyzed data from patients with IBD affected by PSC together with those affected by unrelated diseases such as celiac and eosinophilic enteritis, which may explain some of this difference. Pancolitis was seen in $85 \%$ to $94 \%$ of adults with PSC-IBD versus $45 \%$ to $62 \%$ of adults with UC. ${ }^{2,14,15,16}$ We speculate that this difference is blurred in children because of the higher baseline prevalence of pancolitis in pediatric patients with IBD as a whole, when compared with adults. ${ }^{17}$

\section{Rectal Sparing}

Rectal sparing was present in approximately $20 \%$ of all patients with PSC-IBD and IBD in this study. The reported rates of rectal sparing in PSC-IBD have been 7\% to 52\%.,11,13-15 
Similar to our study, most other series have not found a difference in the rate of rectal sparing between IBD and PSC-IBD. ${ }^{13-15}$

\section{ACTIVITY}

\section{PGA and Mayo Scores}

Clinical and endoscopic activity indices were similar at IBD diagnosis and in follow-up in patients with and without PSC in our study. To our knowledge, this is the first comparison of objective IBD activity indices in pediatric PSC-IBD populations. The initial endoscopy was more often macroscopically normal and showed fewer crypt abscesses on histology in children with UC associated with autoimmune disease versus children with isolated UC in a different study. ${ }^{13}$ Part of this difference may relate to the inclusion of patients with non-PSC with celiac and eosinophilic enteritis in the "autoimmunity" group in that series. A lower histologic inflammatory activity score was seen in patients with PSC-UC (2.09/5) versus patients with UC (2.59/5) in 1 adult series. ${ }^{15}$ The histologic differences may have been more minor than what could be detected with clinical activity scores.

\section{Hospitalizations and Exacerbations}

Patients with IBD and without PSC experienced a greater rate of hospitalization than patients with IBD and PSC, but the difference was not statistically significant. Patients with isolated UC had more disease exacerbations per year ${ }^{13,18,19}$ and more frequent hospitalizations ${ }^{20}$ in other series. We speculate that this difference may relate to the population-based nature of the cohort that our study was drawn from. Our data are likely to include more mild cases of IBD and PSC and therefore is less susceptible to referral bias.

\section{Colectomy}

A lower proportion of patients with PSC-IBD in our study required colectomy compared to patients with IBD and without PSC. This difference did not reach statistical significance however. Similarly, colectomy rates between PSC-IBD and IBD were not significantly different in 3 adult series. ${ }^{14,20,21}$ The overall 5-year rate of colectomy was $24.7 \%$ in IBD and $16.4 \%$ in PSC-IBD, similar to 5-year rates of colectomy of $14 \%$ to $22 \%$ in other pediatric series. $^{22-24}$

\section{RISK FACTORS}

PSC was not a risk factor for colonic surgery in a multivariate regression analysis. We were powered to find a hazard ratio as small as 0.47 (that patients with PSC-IBD were about 2 times less likely to undergo colonic surgery compared to patients with IBD), and smaller differences may exist. We identified male sex and the presence of a comorbid, non-PSC autoimmune disease as risk factors for colonic surgery in patients with IBD. To our knowledge, this was the first time these risk factors have been evaluated in the context of pediatric PSC-IBD. Male sex was previously associated with a greater risk for colectomy in IBD. ${ }^{25}$ Coexistent celiac disease has been associated with more aggressive UC. ${ }^{26}$ Comorbid autoimmune disease has previously been shown to be a risk factor for suboptimal liver outcomes in patients with $\mathrm{AIH}^{27}$ and $\mathrm{PSC},{ }^{28}$ but to our knowledge has not been studied in 
regard to intestinal disease in PSC-IBD. Future analyses of pediatric PSC-IBD activity must account for the higher proportion of men with this phenotype compared to IBD without PSC.

\section{LIMITATIONS}

There were limitations to this study. Because the data are retrospective and dependent on individual clinical practice, not all patients with IBD were screened for PSC, AIH, and/or other autoimmune diseases. The inclusion of undiagnosed patients with PSC may have affected the analyses. Mayo and PGA scores have only 4 possible states and inadequately reflect minor changes in disease activity in individual patients. Due to the rare nature of PSC-IBD, the study was relatively small and we cannot rule out type II error. Small but statistically significant differences may exist between the groups. However, such minor differences in disease activity would not likely be clinically relevant.

\section{CONCLUSIONS}

We compared the IBD distribution and activity in pediatric patients with and without PSC. The prevalence of pancolitis was higher in patients with PSC-IBD than patients with IBD, but the prevalence of rectal sparing disease was similar. IBD activity as measured by the PGA and Mayo endoscopic severity scores, the rate of IBD-related admissions, and the probability of colonic surgery was similar in these patients regardless of the presence of PSC. PSC was not a risk factor for colonic surgery in pediatric IBD in a multivariate regression model. Overall, PSC-IBD did not seem to represent a distinct clinical phenotype of pediatric IBD.

\section{ACKNOWLEDGMENTS}

This investigation was supported by the University of Utah Study Design and Biostatistics Center, with funding in part from the National Center for Research Resources and the National Center for Advancing Translational Sciences, National Institutes of Health, through Grant 5UL1TR001067-02 (formerly 8UL1TR000105 and UL1RR025764).

\section{REFERENCES}

1. Chapman R, Fevery J, Kalloo A, et al. Diagnosis and management of primary sclerosing cholangitis. Hepatology. 2010;51:660-678. [PubMed: 20101749]

2. Loftus EV Jr, Harewood GC, Loftus CG, et al. PSC-IBD: a unique form of inflammatory bowel disease associated with primary sclerosing cholangitis. Gut. 2005;54:91-96. [PubMed: 15591511]

3. Eaton JE, Talwalkar JA, Lazaridis KN, et al. Pathogenesis of primary sclerosing cholangitis and advances in diagnosis and management. Gastroenterology. 2013;145:521-536. [PubMed: 23827861]

4. Nakazawa T, Naitoh I, Hayashi K, et al. Inflammatory bowel disease of primary sclerosing cholangitis: a distinct entity? World J Gastroenterol. 2014;20:3245-3254. [PubMed: 24696608]

5. Deneau M, Jensen MK, Holmen J, et al. Primary sclerosing cholangitis, autoimmune hepatitis, and overlap in Utah children: epidemiology and natural history. Hepatology. 2013;58:1392-1400. [PubMed: 23686586]

6. Dassopoulos T, Nguyen GC, Bitton A, et al. Assessment of reliability and validity of IBD phenotyping within the National Institutes of Diabetes and Digestive and Kidney Diseases (NIDDK) IBD Genetics Consortium (IBDGC). Inflamm Bowel Dis. 2007;13:975-983. [PubMed: 17427244] 
7. Ludwig J Surgical pathology of the syndrome of primary sclerosing cholangitis. Am J Surg Pathol. 1989;13(suppl 1):43-49. [PubMed: 2699167]

8. Desmet VJ. Histopathology of chronic cholestasis and adult ductopenic syndrome. Clin Liver Dis. 1998;2:249-264, viii. [PubMed: 15560031]

9. Rutgeerts P, Sandborn WJ, Feagan BG, et al. Infliximab for induction and maintenance therapy for ulcerative colitis. N Engl J Med. 2005;353: 2462-2476. [PubMed: 16339095]

10. ImproveCareNow. Available at: https://improvecarenow.org/files/media/documents/ Care_Providers/PGA\%20Clinical\%20Guidelines.pdf. Accessed January 5, 2014.

11. Faubion WA Jr, Loftus EV, Sandborn WJ, et al. Pediatric "PSC-IBD": a descriptive report of associated inflammatory bowel disease among pediatric patients with PSC. J Pediatr Gastroenterol Nutr. 2001;33: 296-300. [PubMed: 11593125]

12. Schechter A, Griffiths C, Gana JC, et al. Early endoscopic, laboratory and clinical predictors of poor disease course in paediatric ulcerative colitis. Gut. 2014;64:580-588. [PubMed: 24848266]

13. Ordonez F, Lacaille F, Canioni D, et al. Pediatric ulcerative colitis associated with autoimmune diseases: a distinct form of inflammatory bowel disease? Inflamm Bowel Dis. 2012;18:1809-1817. [PubMed: 22238154]

14. Boonstra K, van Erpecum KJ, van Nieuwkerk KM, et al. Primary sclerosing cholangitis is associated with a distinct phenotype of inflammatory bowel disease. Inflamm Bowel Dis. 2012;18:2270-2276. [PubMed: 22407885]

15. Joo M, Abreu-e-Lima P, Farraye F, et al. Pathologic features of ulcerative colitis in patients with primary sclerosing cholangitis: a case-control study. Am J Surg Pathol. 2009;33:854-862. [PubMed: 19295408]

16. O'Toole A, Alakkari A, Keegan D, et al. Primary sclerosing cholangitis and disease distribution in inflammatory bowel disease. Clin Gastroenterol Hepatol. 2012;10:439-441. [PubMed: 22094024]

17. Ishige $\mathrm{T}$, Tomomasa $\mathrm{T}$, Takebayashi $\mathrm{T}$, et al. Inflammatory bowel disease in children: epidemiological analysis of the nationwide IBD registry in Japan. J Gastroenterol. 2010;45:911917. [PubMed: 20232217]

18. Navaneethan U, Venkatesh PG, Mukewar S, et al. Progressive primary sclerosing cholangitis requiring liver transplantation is associated with reduced need for colectomy in patients with ulcerative colitis. Clin Gastroenterol Hepatol. 2012;10:540-546. [PubMed: 22245961]

19. Marelli L, Xirouchakis E, Kalambokis G, et al. Does the severity of primary sclerosing cholangitis influence the clinical course of associated ulcerative colitis? Gut. 2011;60:1224-1228. [PubMed: 21402617]

20. Lundqvist K, Broome U. Differences in colonic disease activity in patients with ulcerative colitis with and without primary sclerosing cholangitis: a case control study. Dis Colon Rectum. 1997;40:451-456. [PubMed: 9106695]

21. Olsson R, Danielsson A, Järnerot G, et al. Prevalence of primary sclerosing cholangitis in patients with ulcerative colitis. Gastroenterology. 1991; 100:1319-1323. [PubMed: 2013375]

22. Gower-Rousseau C, Dauchet L, Vernier-Massouille G, et al. The natural history of pediatric ulcerative colitis: a population-based cohort study. Am J Gastroenterol. 2009;104:2080-2088. [PubMed: 19436273]

23. Aloi M, D'Arcangelo G, Pofi F, et al. Presenting features and disease course of pediatric ulcerative colitis. J Crohns Colitis. 2013;7: e509-e515. [PubMed: 23583691]

24. Hyams JS, Davis P, Grancher K, et al. Clinical outcome of ulcerative colitis in children. J Pediatr. 1996;129:81-88. [PubMed: 8757566]

25. Chhaya V, Saxena S, Cecil E, et al. The impact of timing and duration of thiopurine treatment on colectomy in ulcerative colitis: a national population-based study of incident cases between 1989 2009. Aliment Pharmacol Ther. 2014;41:87-98. [PubMed: 25382737]

26. Oxford EC, Nguyen DD, Sauk J, et al. Impact of coexistent celiac disease on phenotype and natural history of inflammatory bowel diseases. Am J Gastroenterol. 2013;108:1123-1129. [PubMed: 23419379]

27. Deneau M, Book LS, Guthery SL, et al. Outcome after discontinuation of immunosuppression in children with autoimmune hepatitis: a population-based study. J Pediatr. 2014;164:714-719 e2.

[PubMed: 24423432] 
28. Rupp C, Mummelthei A, Sauer P, et al. Non-IBD immunological diseases are a risk factor for reduced survival in PSC. Liver Int. 2013;33: 86-93. [PubMed: 23157607] 


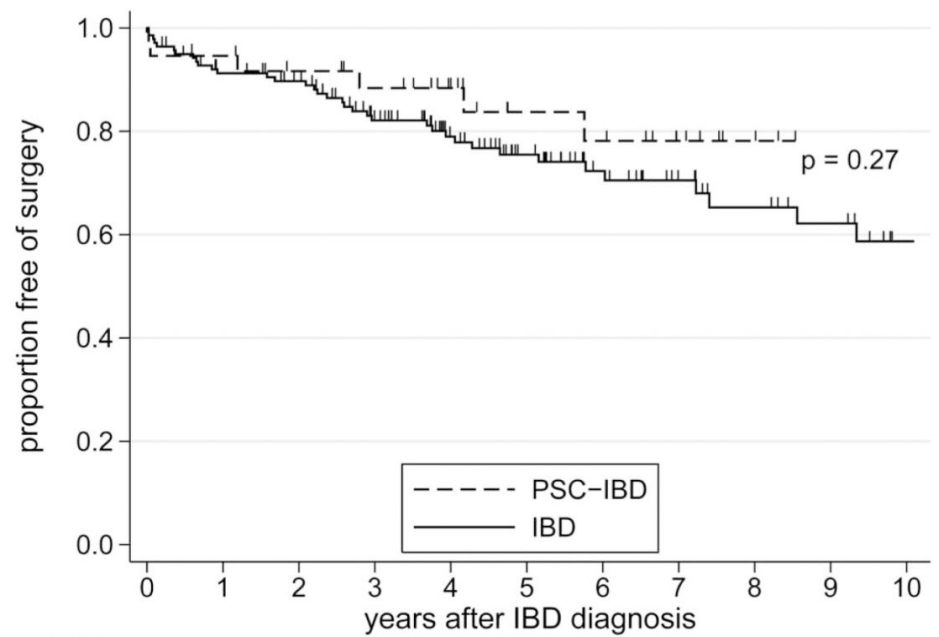

Number at risk

$\begin{array}{cccccccccccc}\text { PSC-IBD } & 37 & 33 & 30 & 27 & 21 & 15 & 14 & 9 & 4 & 2 & 2 \\ \text { IBD } & 139 & 121 & 112 & 90 & 71 & 55 & 40 & 30 & 24 & 20 & 13\end{array}$

FIGURE 1.

Survival free of colonic surgery after diagnosis of inflammatory bowel disease $101 \times 73 \mathrm{~mm}$ $(300 \times 300$ DPI $)$. 
TABLE 1.

Patient Demographics by Diagnosis

\begin{tabular}{lccc}
\hline & PSC-IBD & IBD & $\boldsymbol{P}$ \\
\hline Age at IBD diagnosis, yr & $14.2[9.9-16.7]$ & $14.2[11.7-16.8]$ & 0.431 \\
Follow-up time, yr & $4.75[3.7-7.2]$ & $5.19[3.1-7.8]$ & 0.872 \\
Percent male & $64.9(24 / 37)$ & $56.1(78 / 139)$ & 0.356 \\
UC & $86.5(32 / 37)$ & $88.5(123 / 139)$ & 0.738 \\
Crohn's disease & $13.5(5 / 37)$ & $11.5(16 / 139)$ & 0.738 \\
Pancolitis $^{a}$ & $89.7(26 / 29)$ & $72.4(89 / 123)$ & 0.051 \\
Rectal sparing & $24.3(9 / 37)$ & $21.6(30 / 139)$ & 0.721 \\
Nonhepatic autoimmune disease & $10.8(4 / 37)$ & $6.5(9 / 139)$ & 0.803 \\
AIH overlap & $32.1 \%(9 / 37)$ & - & \\
\hline
\end{tabular}

Data presented as median [interquartile range] or percentage (count) as appropriate.

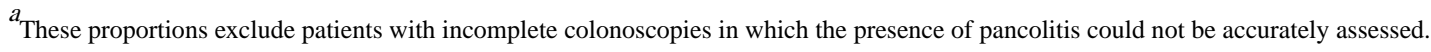




\section{를 \\ 공}

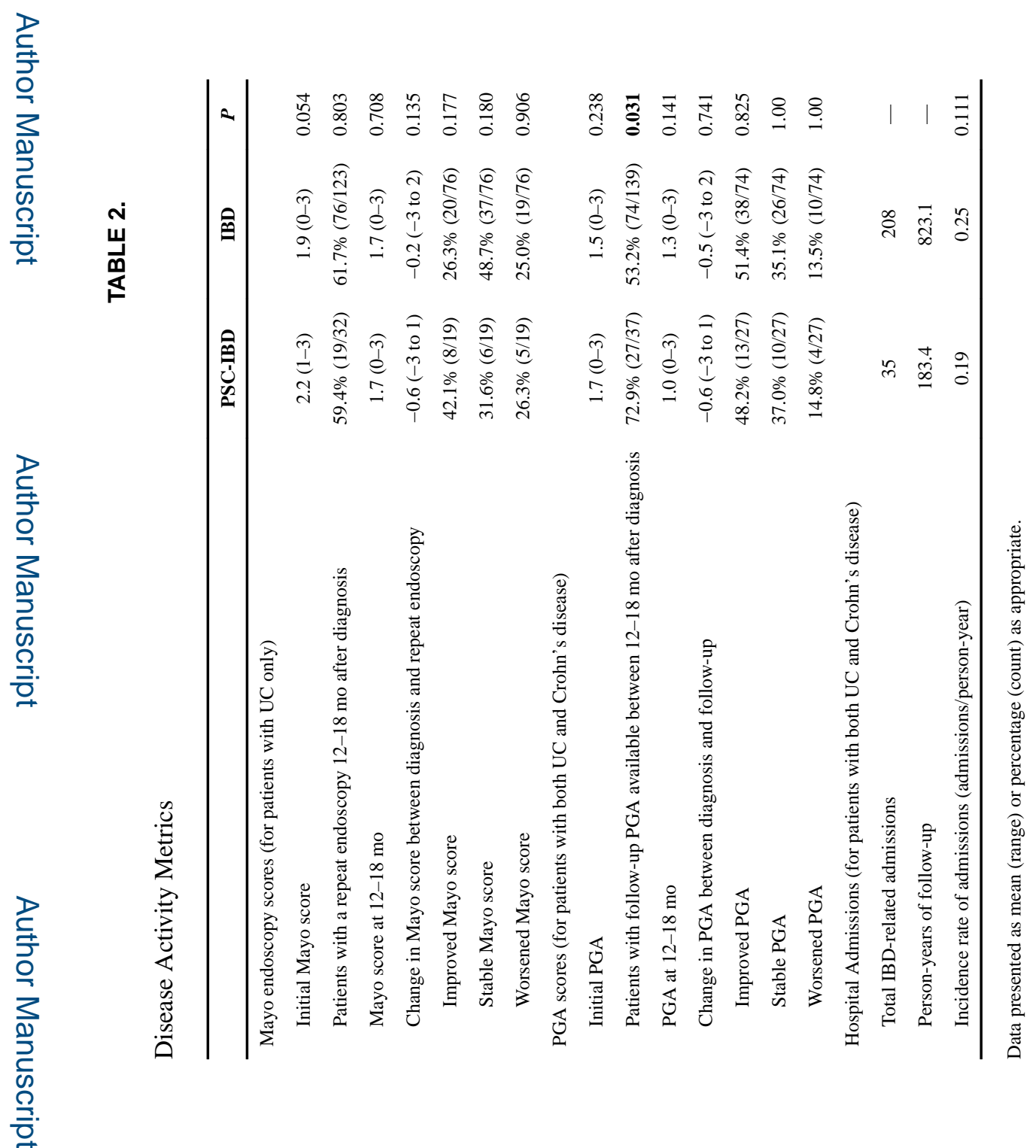

Inflamm Bowel Dis. Author manuscript; available in PMC 2019 April 11. 


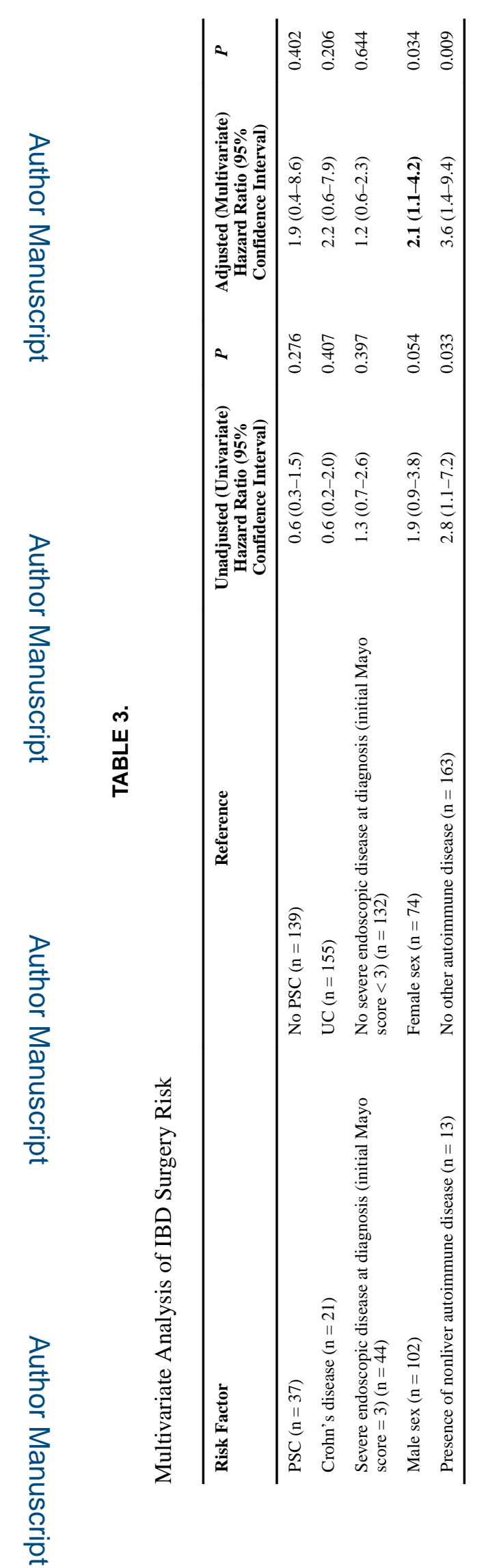

Inflamm Bowel Dis. Author manuscript; available in PMC 2019 April 11. 